\title{
Des modèles animaux très différents pour une même maladie: la glycogénose de type II
}

La glycogénose de type II (GSD II) est une maladie transmise sur le mode autosomique récessif, due au déficit en $\alpha$-glucosidase (GAA). Cette enzyme lysosomale est responsable de l'hydrolyse complète de son substrat naturel, le glycogène, par clivage des liaisons glucosidiques $\alpha-1,4$ et $\alpha-1,6$ à $\mathrm{pH}$ acide, permettant au glucose d'être libéré dans le cytoplasme et d'être réutilisé.

Le déficit en GAA provoque l'accumulation intralysosomale du glycogène dans la plupart des tissus, mais les symptômes cliniques proviennent essentiellement de l'atteinte fonctionnelle des muscles squelettiques et cardiaque. Cliniquement, les nourrissons atteints par la forme précoce de la maladie (forme infantile ou maladie de Pompe) présentent une cardiomyopathie sévère d'évolution fatale avant l'âge de 2 ans. Les patients chez lesquels les premiers signes apparaissent plus tard, pendant l'enfance ou à l'âge adulte (formes infantile ou adulte), ont une myopathie plus ou moins rapidement évolutive, limitée aux muscles squelettiques. Dans ces cas, le décès survient le plus souvent par insuffisance respiratoire. A ce jour, aucune tentative thérapeutique (injections d'enzyme extractive d'origine fungique, greffe de moelle osseuse, transfusion de plasma) ne s'est montrée efficace chez les patients atteints de GSD II.

C'est dire tout l'intérêt que l'on porte aux modèles animaux. Désormais, il en existe trois principaux types:

(1) Il existe en Australie un modèle naturel bovin que ses dimensions physiques et son temps de génération prolongé rendent à l'évidence un modèle peu pratique pour mener des investigations dans un but thérapeutique.

(2) La caille japonaise est également un modèle animal naturel déjà décrit. Kikuchi et al. viennent d'utiliser ce modèle pour tester une approche de thérapie enzymatique substitutive [1]. En effet, ces cailles présentent spontanément des symptômes cliniques : elles ne peuvent pas se relever quand elles sont sur le dos, ni battre des ailes et encore moins voler. Les auteurs ont injecté chez ces animaux de la GAA humaine recombinante par voie intraveineuse. A J18 postinjection, on notait une amélioration des symptômes puisque les oiseaux étaient capables de se relever, de battre des ailes et l'un des animaux a même pu réaliser un vol sur une distance de plus d'un mètre. Parallèlement, l'examen autopsique montrait une augmentation de l'activité enzymatique GAA dans le muscle, associée à une diminution de la surcharge en glycogène. Ces résultats sont assez prometteurs dans la perspective d'une thérapie substitutive chez l'homme qui ferait appel à des injections d'enzyme recombinante. Toutefois, on peut émettre certaines réserves: ce type de modèle animal est assez éloigné de l'homme sur le plan de l'évolution, et la forme de la maladie développée correspond à la forme juvénile ou adulte de GSD II, c'est-à-dire à une myopathie progressive sans cardiomégalie et avec la présence d'une activité enzymatique GAA résiduelle dans les tissus. Ce dernier point pourrait être gênant pour tester l'efficacité d'une approche thérapeutique, qu'il s'agisse de thérapie enzymatique ou génique. (3) Récemment, l'équipe d'Arnold Reuser à Rotterdam vient d'obtenir un modèle murin de GSD II par invalidation du gène de la GAA [2]. Ces souris développent très précocement une surcharge généralisée en glycogène conforme à celle observée dans la forme infantile. Dès 8 jours de vie, on observe au niveau du muscle squelettique une surcharge en glycogène à l'intérieur des lysosomes, sous la forme de granules sombres après coloration spécifique. Cette sur- charge est confirmée par microscopie électronique et évolue progressivement vers le développement de larges vacuoles caractéristiques de la maladie correspondant à des lysosomes bourrés de glycogène non dégradé. La surcharge glycogénique est également retrouvée au niveau du myocarde. Aussi bien dans le muscle squelettique que cardiaque, on observe à 13 semaines de vie une perte de la structure organisée du tissu musculaire. La surcharge en glycogène est également observée dans le foie, le rein, le système nerveux central et le muscle lisse. En revanche, alors que les premiers animaux obtenus sont maintenant âgés de 9 mois, on n'observe pas de symptôme clinique patent (faiblesse musculaire, hypotonie, insuffisance respiratoire ou cardiaque) mais uniquement des signes indirects de cardiopathie: l'électrocardiogramme est pathologique et tout à fait superposable à celui que l'on observe chez les nourrissons atteints (hypertrophie biventriculaire). A l'autopsie, le cœur est très nettement augmenté de volume avec une dilatation très nette des deux ventricules. Les auteurs s'attendent à observer des symptômes cliniques à partir de 12 mois de vie chez ces souris qui vont constituer des outils précieux, non seulement pour explorer l'efficacité de différentes interventions thérapeutiques mais aussi pour mieux comprendre les mécanismes pathogéniques de la GSD II.

M.N.

1. Kikuchi T, Wen Yang H, Pennybacker M, Ichihara N, Mizutani M, Van Hove JLK, Chen YT. Clinical and metabolic correction of Pompe disease by enzyme therapy in acid maltase-deficient quail. J Clin Invest 1998; 101 : 827-33.

2. Bijvoet AGA, Van de Kamp EHM, Kroos MA, Ding JH, Yang BZ, Visser P, Bakker CE, Verbeet MP, Oostra BA, Reuser AJJ, Van der Ploeg AT. Generalized glycogen storage and cardiomegaly in a knockout mouse model of Pompe disease. Hum Mol Genet 1998; 7 : 55-62. 